\title{
Pirogoff's Amputation after Shotgun Injury of the Foot: A case report
}

by Oestern, S. ${ }^{1}$ 国, Trompetter, R. ${ }^{2}$, Lippross, S. ${ }^{1}$, Daniels, M..$^{1}$, Varoga, D. ${ }^{1}$, Mailänder, P. ${ }^{3}$, Weuster, M. ${ }^{1}$, Klüter T. ${ }^{1}$, Seekamp, A. ${ }^{4}$

The Foot \& Ankle Journal 1 (10): 1

Introduction: A gunshot injury can cause extended soft-tissue injury and traumatic contamination leading to infection. The aim of the Pirogoff amputation is to eliminate infection and remove as little of the extremity as possible. This will result in a functional stump with tissues capable of tolerating weight-bearing stress.

Case report: We present a case of a 73-year-old male with extended soft-tissue injury of the left foot and multiple pellets in the surrounding tissues after shotgun injury. Aside from arterial hypertension and noninsulin-dependent diabetes mellitus, the patient suffered from obesity and peripheral peroneal paresis after thromboembolectomy in 1997. Systemic and local examination showed no neurological or vascular compromise to the foot. Radiographs revealed comminuted fractures of the talus, navicular and medial cuneiform. A Priogoff amputation was chosen as the amputation of choice.

Conclusion: When higher amputation of the foot is necessary, Pirogoff's amputation is a useful procedure and offers satisfying results in patients without vascular defects. This amputation preserves more of the limb than a below-the-knee amputation and appears more functional and resilient than a Syme's amputation. It also provides a lesser degree of limb length discrepancy than the Syme's amputation.

Key words: Pirogoff's amputation, tibia-calcaneal arthrodesis, shotgun injury reproduction in any medium, provided the original work is properly cited. @The Foot \& Ankle Journal (www.faoj.org)

Irreversible destruction of the forefoot and midfoot generally leads to amputation. Transmetatarsal, Chopart or Syme's amputations can often result in poor clinical outcomes. ${ }^{4}$

Address correspondence to: Dr. Stefanie Oestern. Department of Trauma Surgery, University of Schleswig-Holstein, Campus Kiel. Arnold-HellerStraße 3, 24105 Kiel, Germany Email: Stefanie.Oestern@uksh-kiel.de

${ }^{1}$ Resident of Trauma Surgery, UK-Schleswig-Holstein, Campus Kiel, Germany.

${ }^{2}$ Senior Physician of Trauma Surgery, UK-Schleswig-Holstein, Campus

Kiel, Germany.

${ }^{3}$ Chief of Plastic Surgery, UK-Schleswig-Holstein, Lübeck, Germany.

${ }^{4}$ Chief of Trauma Surgery, UK-Schleswig-Holstein, Campus Kiel, Germany.
Prostheses for such stumps can be difficult to fit. This reduces the mobility of the patient and reamputations are sometimes necessary. ${ }^{4}$

Pirogoff's arthrodesis was first described by Pirogoff in 1854. ${ }^{4}$ It can serve as a surgical salvage procedure in complex injuries of the forefoot where considerable osseous and soft tissue defects are present. This paper presents a case report of gunshot injury to the left foot. 


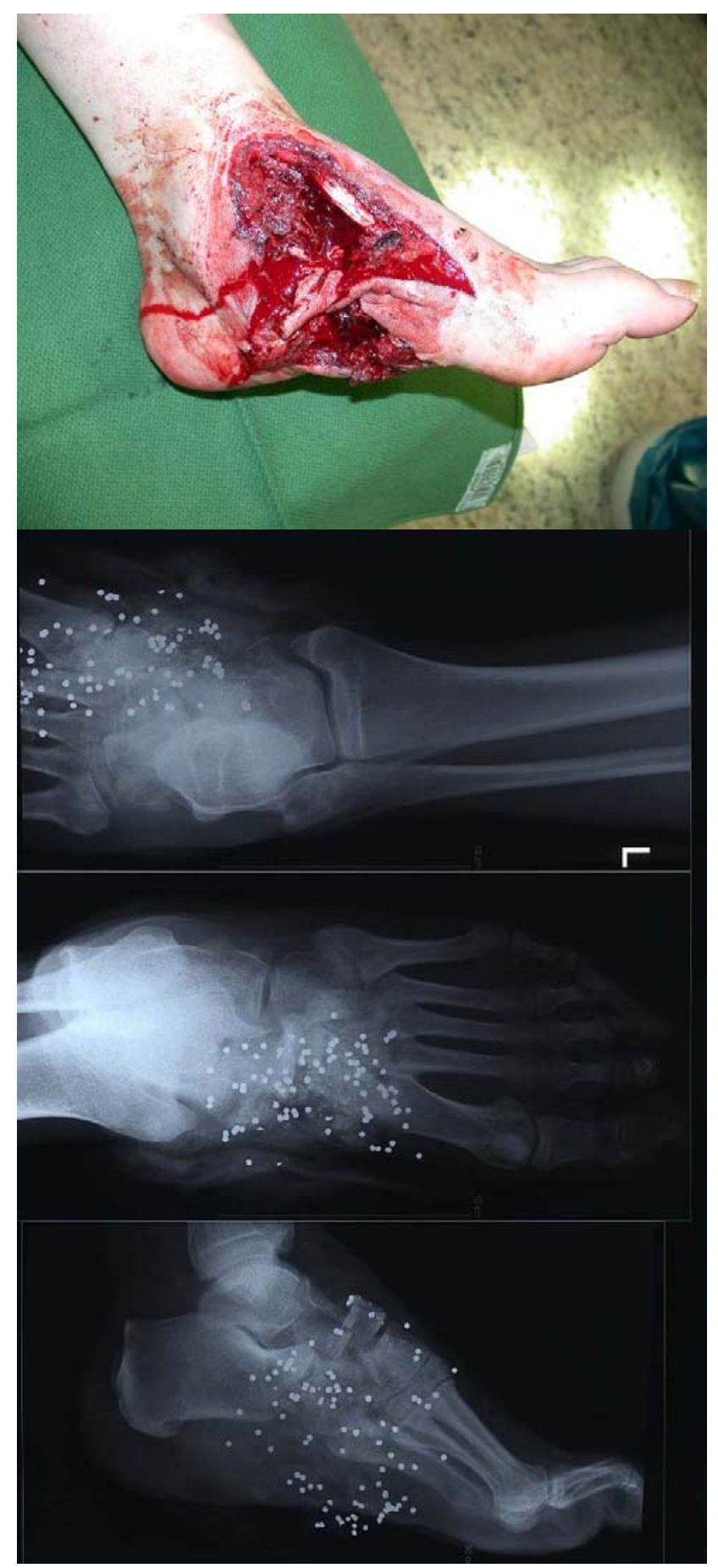

Figure 1AB Shotgun injury of the left foot with extended soft-tissue injury. Multiple pellets in surrounded tissue is shown on radiograph.

\section{Case presentation}

A 73 year-old male suffered a shotgun injury to the left foot while loading a shotgun. He sustained extensive soft-tissue injury to the medial aspect of the mid foot and comminuted fractures of the talus, navicular and medial cuneiform. (Figs.1AB)

The patient had the following comorbidities: morbid obesity, arterial hypertension, and noninsulin-dependent diabetes mellitus. After thrombembolectomy in 1997, the patient regularly took warfarin. The patient was referred to the author`s trauma center. Initial digital angiography showed an occlusion of the anterior tibial artery and perfusion of the posterior tibial and peroneal arteries.

On the day of injury, a wound debridement was performed and the foot was stabilized by external fixation. Two days later, a vacuum assisted closure therapy was started. Due to the extensive softtissue defect and destruction of the medial column of left foot, Pirogoff's forefoot amputation was performed by rotation of the dorsal part of calcaneus to 70 degrees. This was performed in cooperation with the department of plastic surgery at the University of SchleswigHolstein Campus, Lübeck, Germany. (Figs. 2AB)

The wound defect was covered with a dorsalis pedis flap. Three days later, the drain was removed. Apart from a postoperative hematoma, the operation proceeded without complication. One month after operative treatment, the patient was referred to another hospital for functional rehabilitation. Three months after amputation the patient was fitted with a prothesis. (Fig. 3) 


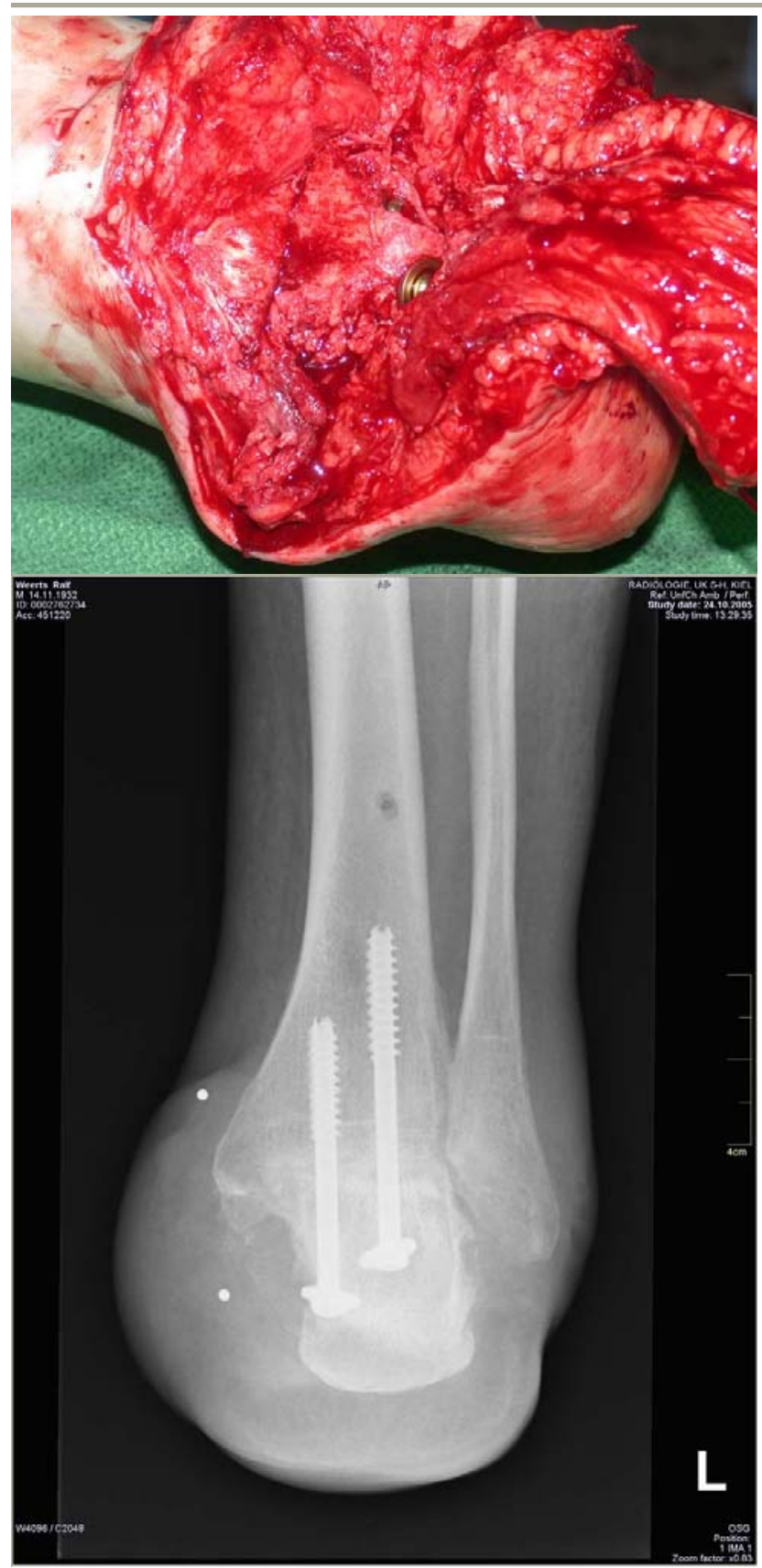

Figure 2AB Pirogoff's forefoot amputation was performed by rotation of dorsal part of calcaneus up to 70 degrees and fused to the tibial plafond.

\section{Discussion}

Severe injuries require a coordinated approach in treatment. Goals of this treatment are to assure functional limb salvage. ${ }^{1}$ When determining the level of amputation, comorbidities such as diabetes and occlusive vascular disease should be considered.

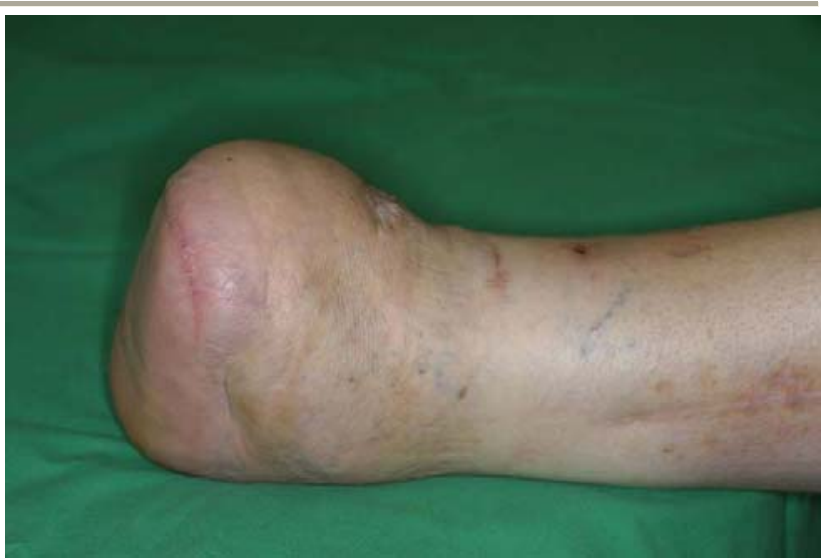

Figure 3 The patient was fitted with a prothesis three months after the Pirogoff amputation.

Gunshot wounds, as in the described case, are frequently accompanied by extended soft-tissue infection and fractures. Compartment syndromes should not be overlooked in open fractures as the adjacent compartments are still at risk. A compartment syndrome can communicate with the lower leg and can compromise limb salvage. A low threshold for fasciotomy should be maintained in these complicated injuries. A compartment syndrome can communicate with the lower leg and can compromise limb salvage.

Large crush injuries, as is often seen with shotgun wounds, are also predisposed to higher rates of infection due to the concomitant muscle hypoxia and secondary necrosis. ${ }^{1}$ An analysis of outcomes of reconstruction or amputation after legthreatening injuries showed that the outcomes among patients who underwent reconstruction were not significantly different from those patients who underwent amputation. ${ }^{2}$ Irreversible destruction of the forefoot and midfoot with concomitant wound contamination generally leads to amputation.

The decision to amputate may be immediate within the first 24 hours or delayed as a procedure within the first hospitalization. Several authors have developed scoring algorithms to help guide this decision. ${ }^{3,6}$ However, reconstruction compared to amputation is associated with a higher risk of complication, additional surgical treatment and rehospitalization. 
Before operative treatment, sufficient diagnostics and imaging must be performed. A retrospective review was undertaken by Odland to determine risk factors associated with amputation after open fractures of the lower extremity that were complicated by vascular injury. ${ }^{8}$ The prognosis remains unfavorable for the patient with polytrauma who is admitted with a crushed limb in shock. The degree and length of ischemia are critical factors that should be addressed. Vascular repair, debridement of devitalized tissue and stabilization of the bone is essential for successful outcome. $^{8}$

In this case, digital angiography revealed good perfusion of the posterior tibial and peroneal arteries. The anterior tibial artery showed an occlusion which was not of overriding importance for the blood supply to the calcaneus. Some studies have shown that Lisfranc, Chopart and Syme's amputations are associated with wound healing complications and the need for revisional amputation. ${ }^{5,7}$ Therefore, the indication on level of amputation should to be carefully considered. ${ }^{9}$ Prostheses after transmetatarsal amputation are also difficult to fit. The mobility of these patients is sometimes reduced and further revision may be necessary. ${ }^{4}$

The tibiocalcaneal arthrodesis introduced by Pirogoff in 1854 can be a useful surgical alternative in selected cases. ${ }^{4}$ The Pirogoff ankle disarticulation maintains limb length by rotating the calcaneus and does not cause severe pain because of the existence of the calcaneus and fat pad for weightbearing. ${ }^{10}$

Injuries limited to the forefoot, especially in shotgun wounds, when reconstruction of the forefoot is not possible are an indication for forefoot amputation with arthrodesis between tibia and calcaneus. Absolute contraindications to the Pirogoff's arthrodesis are the destruction of the calcaneus, osteomyelitis of the lower tibia and calcaneus and malignancy of the heel. ${ }^{11}$
The Pirogoff amputation offers a more stable stump and higher mobility compared to the Syme's amputation. ${ }^{11}$ The healed flap is less vulnerable than in a Syme's amputation and the stump offers a minimum of limb length shortening. This fascilitates a better fit of the stump into a prothesis and allows some limited barefoot mobility.

Deep infection or osteoporosis may delay or prevent fusion between tibia and calcaneus. This seems to be one of the most important considerations when making a decision to perform the Pirogoff arthrodesis.

However, difficulties such as bony fixation and healing of the arthrodesis can be minimized by using an external ring fixation system.

\section{Conclusion}

Large zone crush injuries are predisposed to high rates of infection due to muscle hypoxia and secondary necrosis. Therefore, the procedure of choice in many of these cases is amputation. When ankle disarticulation is necessary, surgeons have various options. The Syme amputation can result in a leg-length discrepancy and Chopart's amputation may lead to dropfoot, which can compromise walking. ${ }^{10}$

The Pirogoff ankle disarticulation can be a viable procedure alternative. Our case, therefore, recommends Pirogoff amputation in patients with irreversible destruction of the forefoot and midfoot. It maintains leg length by rotating the calcaneus. Comorbidities and other factors such as age and risk of infection should however be considered before surgery. 


\section{References}

1. Agnew S: Lower Extremity Limb Salvage: Decision Making \& Technical Challenges. Jacksonville Medicine, 1998. [online]

2. Bosse MJ, MacKenzie, EJ, Kellum, JF, et al.: An analysis of outcomes reconstruction or amputation after legthreatening injuries. The New England Journal of Medicine: 347: 1924-1931, 2002.

3. Dirschl DR, Dabners LE: The mangled extremity: when should it be amputated? Journal of American Academy of Orthopaedic Surgeons: (Volume 4) 182-190, 1996.

4. Einsiedel T, Dieterich J, Kinzl L et al: Lower limb salvage using Pirogoff ankle arthrodesis: Minimally invasive and effective fixation with the Ilizarov external ring fixator. Orthopäde 37(2): 143-152, February 2008.

5. Krieghoff R: The Pirogoff amputation from the current viewpoint. Beit Orthop Traumatologie: 29(8): 434-438, August 1982.

6. Lange RH: Limb reconstruction vs. Amputation decision making in massive lower extremity trauma. Clinical Orthopaedics: Volume 243: 92-99, 1989.

7. Pollard J. Hamilton S, Rush S et al: Mortality and morbidity after transmetatarsal amputation: retrospective review of 101 cases. The Journal of Foot \& Ankle Surgery: 45(2): 91-97, March, 2006.

8. Odland MD, Gisbert VL, Gustilo RB et al: Combined orthopedic and vascular injury in the lower extremities: indications for amputation. Surgery; 108(4): 664-666, 1980. 9. Siev-Ner I, Heim M, Warshawski M et al: A review of the aetiological factors and results of trans-ankle (Syme)

disarticulations. Disability \& Rehabilitation: 28(4): 239-242, February, 2006.

10. Taniguchi A, Tanaka Y, Kadono K et al: Pirogoff ankle disarticulation as an option for ankle disarticulation. Clinical Orthopaedics and related research: 414: 322-328, 2003.

11. Warren G. Conservative Amputation of the neuropathic Foot-The Pirogoff Procedure. Operative Orthopädie und Traumatologie: 9 (1): 49-58, March, 1997. 